\title{
Predictive value of naitfold capillaroscopy in patients with Raynaud's phenomenon
}

Meli, Madeleine ; Gitzelmann, Gabriela ; Koppensteiner, Renate ; Amann-Vesti, Beatrice R

\begin{abstract}
The objective of this study was to evaluate the long-term follow-up of patients with Raynaud's phenomenon (RP) and pathological nailfold capillaroscopy (NC) in order to analyse the predictive value of specific features of capillaroscopy for the development of a connective tissue disease (CTD). From 1992 to 2002, NC alone or combined with fluorescence videomicroscopy with sodium fluorescein (NaF) was performed in 1024 consecutive patients because of RP. We analysed the follow-up and pathological features of NC in all patients who had neither clinical nor serological signs of a CTD at the time of NC. Of 308 patients with neither serological findings nor clinical signs of CTD but with RP and pathological features in NC suspicious for CTD, follow-up data were available for 133 patients. An additional NaF test had been performed in 51 (38.4\%) patients. After a mean follow-up of 6.5 years (range: 1-15 years), 109 patients had developed a CTD and 24 patients did not show any clinical signs or serological markers for a CTD after a mean follow-up of 8.5 years (range: $2-15$ years). There were no differences in age, duration of RP or of follow-up in patients who developed a CTD compared to patients who did not. Significantly more giant capillaries $(\mathrm{p}=0.0001)$, avascular fields $(\mathrm{p}=0.02)$ and irregular architecture $(\mathrm{p}=0.0001)$ had been observed in patients who had developed a CTD during the follow-up of 6.5 years. The presence of giant capillaries, avascular fields and irregular architecture of nailfold capillaries is predictive for the development of a CTD in patients with RP
\end{abstract}

DOI: https://doi.org/10.1007/s10067-005-1146-1

Posted at the Zurich Open Repository and Archive, University of Zurich

ZORA URL: https://doi.org/10.5167/uzh-155999

Journal Article

Published Version

Originally published at:

Meli, Madeleine; Gitzelmann, Gabriela; Koppensteiner, Renate; Amann-Vesti, Beatrice R (2006). Predictive value of naitfold capillaroscopy in patients with Raynaud's phenomenon. Clinical Rheumatology, 25(2):153-158.

DOI: https://doi.org/10.1007/s10067-005-1146-1 


\section{Predictive value of nailfold capillaroscopy in patients with Raynaud's phenomenon}

Received: 16 November 2004/ Revised: 23 March 2005/ Accepted: 23 March 2005/Published online: 11 June 2005

(C) Clinical Rheumatology 2005

\begin{abstract}
The objective of this study was to evaluate the long-term follow-up of patients with Raynaud's phenomenon (RP) and pathological nailfold capillaroscopy (NC) in order to analyse the predictive value of specific features of capillaroscopy for the development of a connective tissue disease (CTD). From 1992 to 2002, NC alone or combined with fluorescence videomicroscopy with sodium fluorescein $(\mathrm{NaF})$ was performed in 1024 consecutive patients because of RP. We analysed the follow-up and pathological features of NC in all patients who had neither clinical nor serological signs of a CTD at the time of NC. Of 308 patients with neither serological findings nor clinical signs of CTD but with RP and pathological features in NC suspicious for CTD, follow-up data were available for 133 patients. An additional $\mathrm{NaF}$ test had been performed in $51(38.4 \%)$ patients. After a mean follow-up of 6.5 years (range: 1-15 years), 109 patients had developed a CTD and 24 patients did not show any clinical signs or serological markers for a CTD after a mean follow-up of 8.5 years (range: 2-15 years). There were no differences in age, duration of RP or of follow-up in patients who developed a CTD compared to patients who did not. Significantly more giant capillaries $(p=0.0001)$, avascular fields $(p=0.02)$ and irregular architecture $(p=0.0001)$ had been observed in patients who had developed a CTD during the follow-up of 6.5 years. The presence of giant capillaries, avascular fields and irregular architecture of nailfold capillaries is predictive for the development of a CTD in patients with RP.
\end{abstract}

M. Meli · G. Gitzelmann

R. Koppensteiner · B. R. Amann-Vesti ( $₫)$

Division of Angiology, Department of Internal Medicine,

University Hospital, Ramistrasse 100,

8091 Zurich, Switzerland

E-mail: beatrice.amann@usz.ch

Tel.: + 41-1-2551111

Fax: + 41-1-2554510
Keywords Connective tissue disease $\cdot$ Nailfold capillaroscopy · Raynaud's phenomenon - Systemic sclerosis

\section{Introduction}

Raynaud's phenomenon (RP) is classified as primary when no underlying cause can be identified and as secondary when its presence is explained by an associated condition such as systemic lupus erythematosus (SLE) and systemic sclerosis. Estimates of the prevalence of RP in the general population range from 5 to $20 \%$ [1], and $3-5 \%$ have been reported to develop a connective tissue disease (CTD) within 3-6 years [2]. In scleroderma, RP has been reported to be the presenting sign in $50-70 \%$ of patients and in $15 \%$ of patients with SLE [3-5]. However, RP may precede the development of the disease by many years. In several small studies it has been reported that the presence of autoantibodies may predict the presence or future development of systemic disease [6-8].

Nailfold capillaroscopy (NC) allows the in vivo assessment of morphology and of some functional aspects of cutaneous capillaries and has been accepted as a diagnostic tool for evaluating microvascular involvement in systemic sclerosis, dermatomyositis, overlap syndromes and SLE [9-12]. Only little is known about the role of NC in identifying patients with RP who are at risk of developing a CTD [13-15]. Furthermore, by fluorescence videomicroscopy with sodium fluorescein $(\mathrm{NaF})$ patients with a CTD might exhibit halo enlargement with a typical "dwarf hat" formation as a sign of disturbed barrier function of the vessel wall and structural changes of the pericapillary space [16]. However, it has been shown in one study that of 69 patients with CTD $16 \%$ had normal findings in conventional NC but pathological halo formation after $\mathrm{NaF}$ injection [17]. The predictive value of combined $\mathrm{NC}$ with $\mathrm{NaF}$ has not been studied yet. In the present report, we describe the followup of 133 patients with pathological findings in NC with 
or without $\mathrm{NaF}$ who were referred to our clinic because of RP. At the time of the study, all patients had negative autoantibodies and no other clinical signs of a CTD.

\section{Patients and methods}

In the period from 1992 to 2002,1024 patients were referred to our institution for vascular assessment including NC because of a history of RP of the hands and fingers. In all patients NC was performed; fluorescence videomicroscopy with $\mathrm{NaF}$ was only performed in part of the patients. Exclusion criteria for $\mathrm{NaF}$ were drug or food allergies, history of renal disease, pathological creatinine clearance, and pregnancy. In some cases the $\mathrm{NaF}$ test was not performed because of difficulties in puncturing a vein or no specific reason was given. Fluorescence videomicroscopy with $\mathrm{NaF}$ was performed as previously described [18]. NC was performed with a fluorescence videomicroscopy system consisting of an incident light fluorescence microscope (Leica, Heerbrugg, Switzerland), a 3-CCD video camera (model DXC930P, Sony, Tokyo, Japan) with a camera adapter and sensitivity set on automatic control (CMA-D2, Sony), a video timer (VTG-22) and scale marker (IV-600, both from For-A-Company, Tokyo, Japan), a video monitor (Picture Monitor model PM 171T, Ikegami Tsushinki, Tokyo, Japan) and a video tape recorder (S-VHS, AG7350, Panasonic, Osaka, Japan). The microscope is equipped with $1.0 / 0.04,2.5 / 0.08,6.3 / 0.20$ and $10 / 0.25$ planar objectives (Leica, Heerbrugg, Switzerland), which allow a magnification of 24, 62, 165 and 240 times, respectively, on the monitor. The fluorescence excitation filter works at $450-490 \mathrm{~nm}$ and the barrier filter at $515 \mathrm{~nm}$. The $\mathrm{NaF}$ was injected into the antecubital vein as a bolus; the dosage was adjusted according to the estimated blood volume. At our institution $0.2-0.3 \mathrm{ml}$ $\mathrm{NaF} 20 \%$ per liter estimated blood volume is used and is adequate for visualization of skin capillaries.

The following parameters of $\mathrm{NC}$ were evaluated from the videotape [19]:

- Irregular architecture (capillaries not in one row as normal, small areas $(<500 \mu \mathrm{m})$ with missing capillaries next to areas with clusters of capillaries)

- Avascular fields (loss of capillaries in a field of at least $500 \mu \mathrm{m})$

- Dilatation of capillaries (arterial side $>15 \mu \mathrm{m}$, venous side $>20 \mu \mathrm{m}$ )

- Giant capillaries (apical diameter $>50 \mu \mathrm{m}$, Fig. 1)

- NaF images were evaluated for apical halo enlargement or "dwarf hat" formation, respectively (Fig. 2) [16].

\section{Diagnostic criteria}

The diagnosis of systemic sclerosis (Scl) was made according to the preliminary criteria of the American

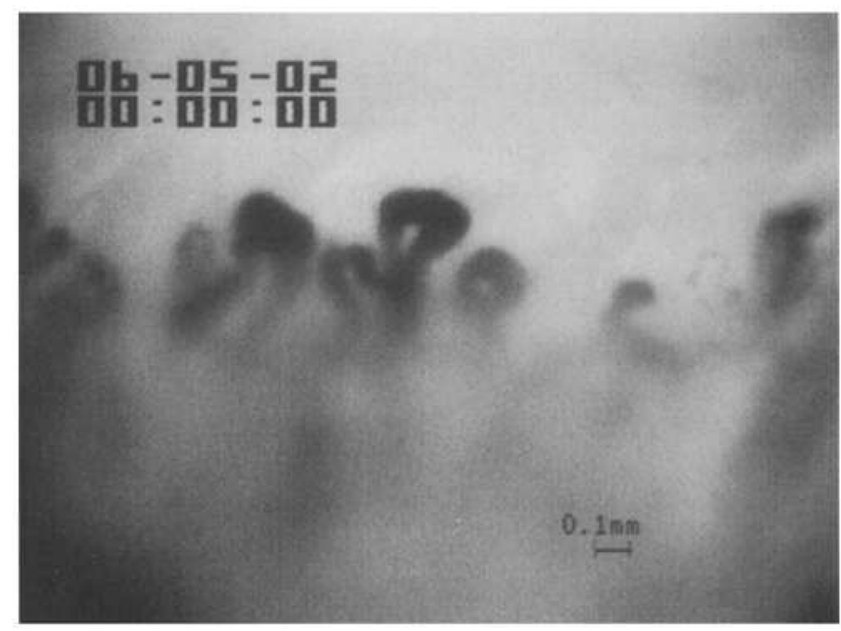

Fig. 1 Typical giant capillary of the nailfold in a female patient with RP who developed CREST 10 years after NC $(\times 63)$.

Rheumatism Association (ARA) [20]. The diagnosis of CREST was made when the following criteria were fulfilled: calcinosis, Raynaud's phenomenon, oesophageal dysmotility, sclerodactyly, and telangiectasia in the
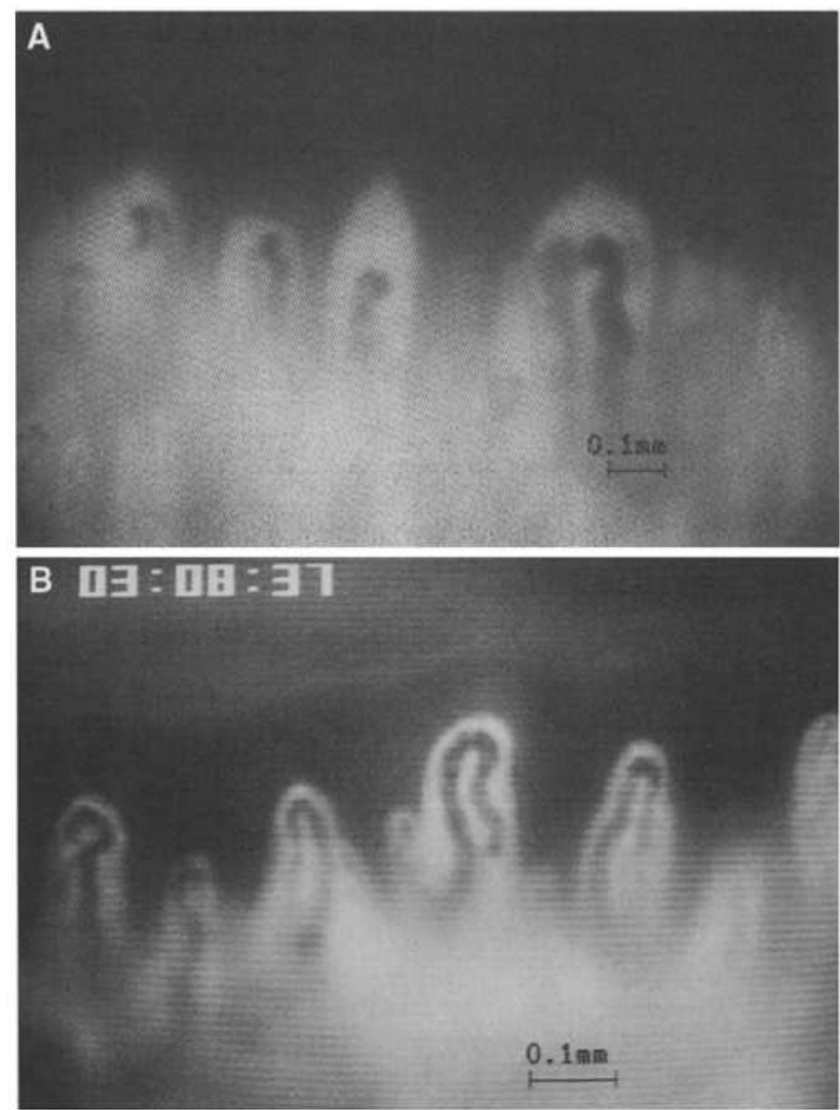

Fig. 2 a After the intravenous injection of $\mathrm{NaF}$ apical halo enlargement or "dwarf hat" formation might be seen in patients with CTD. b In healthy subjects the cells are surrounded by the fluorescent plasma layer and $\mathrm{NaF}$ does not pass the narrow pericapillary border $(\times 63)$. 
presence of anticentromere antibodies. Mixed connective tissue disease (MCTD) was diagnosed in the presence of symptoms as described by Alarcon-Segovia and Cardiel [21]. The diagnoses of SLE and rheumatoid arthritis (RA) were based on the diagnostic criteria of the ARA. Dermatomyositis (DM) was considered the diagnosis when the criteria of Bohan and Peter [22] were present. Sjögren's syndrome was diagnosed when the diagnostic criteria from the American-European Consensus Group were present, including the presence of autoantibodies (anti-Ro/SSA, anti-La/SSB or both) [23].

\section{Statistical analysis}

Analyses were performed with the statistical software package Stat View 5.0. Continuous variables are reported as means and categorical variables as percentages. Comparison between groups of patients was done by means of unpaired Student's $t$-test and for categorical variables by using Fisher's exact test. A test for logistic regression was performed as a means of studying the diagnostic value of the different features of $\mathrm{NC}$ to predict development of CTD. Significance was defined as $p<0.05$.

\section{Results}

Of a total of 1024 patients seen with RP between 1992 and 2002, the results of 968 patients were evaluated for the present study; 19 patients with Osler's disease and 37 patients for whom some data were missing had been excluded. In 693 patients, the $\mathrm{NaF}$ test was performed in addition to conventional NC. In 201 (155 females and 46 males) patients referred to us because of RP the diagnosis of a CTD had already been made at the time of NC due to serological features and the above-mentioned criteria. The diagnosis and results of NC from these 201 patients are shown in Table 1. Microangiopathy was defined as being present if at least one of the abovementioned criteria was found. The diagnosis of primary RP was made in 459 patients because the following criteria were fulfilled: normal findings in $\mathrm{NC}$, negative autoantibodies, no signs of a CTD according to the
ARA criteria, no calcinosis, no oesophageal motility disturbances, no sclerodactyly, no telangiectasia and no xerostomia. In 308 patients with normal serological findings and no clinical signs of a CTD despite RP, we found pathological features in NC suspicious for CTD. Of these 308 patients with RP and microangiopathy in NC, follow-up data were available for 133 patients: 107 females (mean age: 50.0 years, range: $10-89$ years) and 26 males (mean age: 55.1 years, range: $26-82$ years). The $\mathrm{NaF}$ had been administered in $51(38.4 \%)$ patients. After a mean follow-up of 6.5 years (range: $1-15$ years), 109 patients (82\%, group 1) had developed a CTD according to the above-described criteria. The patient characteristics, diagnoses and findings of $\mathrm{NC}$ in these patients are shown in Table 2.

The remaining 24 patients (group 2, 19 females, 5 males, mean age: 45.6 years, range: $21-70$ ) had not developed any clinical signs of a CTD and serological tests were still negative after a mean follow-up of 8.5 years (range: $2-15$ years). Patient characteristics and features of NC are shown in Table 3 . There were no statistically significant differences in age, duration of RP or duration of follow-up in patients who developed a CTD (group 1) compared to patients who had not developed a CTD (group 2) during the follow-up. For comparison between the two groups, only patients who had not developed a CTD after a follow-up of at least 6 years had been included: four patients with a followup of 4 years (two patients), one patient with a followup of 5 years and one patient with a follow-up of 2 years had been excluded from the analysis [2]. The mean follow-up of these 20 patients was 9.3 years (range: 6 15 years). The features and comparison of NC in the two groups are shown in Fig. 3. In group 1 significantly more giant capillaries $(p=0.0001)$, avascular fields $(p=0.02)$ and irregular architecture $(p=0.0001)$ had been observed at the initial investigation. Including all 24 patients the results were almost identical; the same features (giant capillaries, avascular fields and irregular architecture) had been identified as significantly different in the two groups $(p<0.05)$.

Furthermore, the presence of giant capillaries or an irregular architecture was predictive for the development of a CTD $(p<0.005)$. Only two patients developed SLE
Table 1 Age, sex and presence or absence of pathological features in nailfold capillaroscopy (NC) of 201 patients with RP, in whom an underlying disease had been already diagnosed at the time of NC. $S c l$ systemic sclerosis, CREST calcinosis, Raynaud's phenomenon, oesophageal dysfunction, sclerodactyly, telangiecstasia, $S L E$ systemic lupus erythematosus, $M C T D$ mixed connective tissue disease, $D M$ dermatomyositis, $R A$ rheumatoid arthritis

\begin{tabular}{llllll}
\hline Diagnosis & $\begin{array}{l}\text { Patients } \\
(n / \%)\end{array}$ & $\begin{array}{l}\text { Age (mean, } \\
\text { range, years) }\end{array}$ & $\begin{array}{l}\text { Female/male } \\
(\%)\end{array}$ & $\begin{array}{l}\text { Presence of } \\
\text { microangiopathy } \\
(\%)\end{array}$ \\
\cline { 4 - 5 } & & & Yes & No \\
\hline Scl & $93 / 46.3$ & $52.4(13-78)$ & $72.0 / 28.0$ & 83.9 & 16.1 \\
CREST & $8 / 4.0$ & $62.4(36-76)$ & $87.5 / 12.5$ & 87.5 & 12.5 \\
SLE & $29 / 14.4$ & $39.1(12-63)$ & $82.8 / 17.2$ & 37.9 & 62.1 \\
MCTD & $29 / 14.4$ & $50.7(27-83)$ & $86.2 / 13.8$ & 65.5 & 34.5 \\
DM & $14 / 7.0$ & $52.6(17-70)$ & $57.1 / 42.9$ & 57.1 & 42.9 \\
Sjögren's & $9 / 4.5$ & $52.6(17-69)$ & $88.9 / 11.1$ & 44.5 & 55.5 \\
syndrome & $19 / 9.4$ & $50.3(22-73)$ & $84.2 / 15.8$ & 31.6 & 68.4 \\
RA & 201 & $51.5(12-83)$ & $77.1 / 22.9$ & 60.7 & 39.3 \\
Total & & &
\end{tabular}


Table 2 Characteristics of 109 patients with RP and pathological features in the nailfold capillaroscopy and their diagnosis after a mean follow-up of 6.5 years (1-15 years). Scl systemic sclerosis, CREST calcinosis, Raynaud's phenomenon, oesophageal dys-

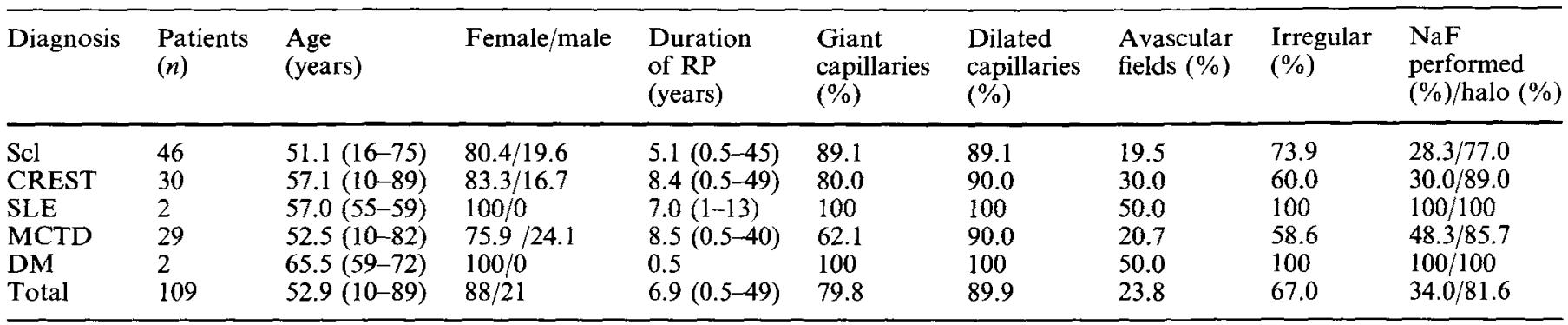

function, sclerodactyly, telangiecstasia, $S L E$ systemic lupus erythematosus, $M C T D$ mixed connective tissue disease, $D M$ dermatomyositis

Table 3 Characteristics of 24 patients with RP and pathological features in the nailfold capillaroscopy who did not develop CTD after a mean follow-up of 8.5 years (2-15 years)

\begin{tabular}{|c|c|c|c|c|c|c|c|c|}
\hline $\begin{array}{l}\text { Patients } \\
\text { (n) }\end{array}$ & $\begin{array}{l}\text { Age } \\
\text { (years) }\end{array}$ & $\begin{array}{l}\text { Female/male } \\
(\%)\end{array}$ & $\begin{array}{l}\text { Duration } \\
\text { of RP (years) }\end{array}$ & $\begin{array}{l}\text { Giant } \\
\text { capillaries } \\
(\%)\end{array}$ & $\begin{array}{l}\text { Dilated } \\
\text { capillaries } \\
(\%)\end{array}$ & $\begin{array}{l}\text { Avascular } \\
\text { fields }(\%)\end{array}$ & $\begin{array}{l}\text { Irregular } \\
(\%)\end{array}$ & $\begin{array}{l}\text { NaF performed } \\
(\%) / \text { halo }(\%)\end{array}$ \\
\hline
\end{tabular}

and DM, respectively; therefore no conclusions for these diseases can be drawn from our data.

\section{Discussion}

NC has been accepted as one of the most valuable diagnostic tools for the early detection of CTD, in particular for Scl and CREST [24-26]. In the present study, we analysed the features of $N C$ in patients with $R P$ in order to evaluate the predictive value of pathological findings. We found that during the mean follow-up of

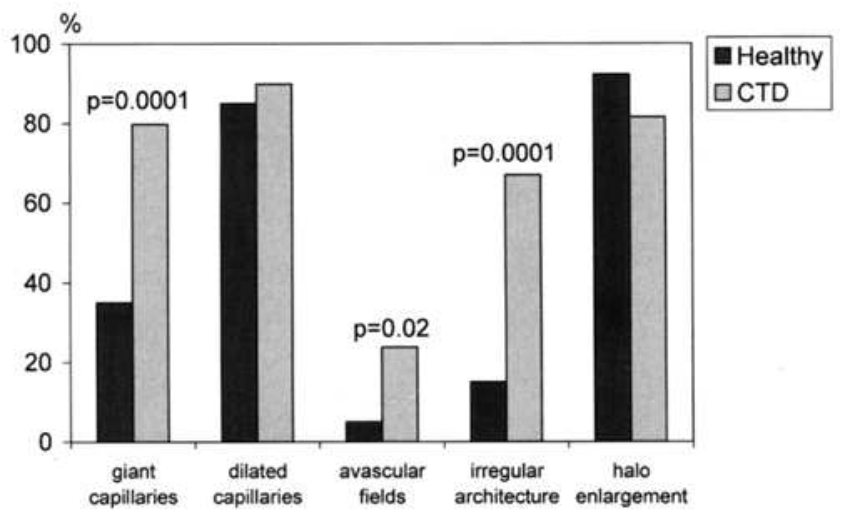

Fig. 3 Comparison of features of nailfold capillaroscopy in patients with RP. In patients who developed a connective tissue disease $(C T D)$ during follow-up significantly more giant capillaries $(p=0.0001)$, avascular fields $(p=0.02)$ and irregular architecture $(p=0.0001)$ have been found than in the 20 patients without any laboratory or clinical signs of a CTD after a follow-up of at least 6 years (mean follow-up: 9.3 years, range: 6-15 years). The enlargement of the apical halo after injection of $\mathrm{NaF}$ was not different in the two groups. 
It is known that a CTD might develop many years after the onset of RP; therefore, the Allen and Brown criteria requiring at least a 2-year history of RP for the diagnosis of a primary RP have been revised [30]. In our analysis the mean duration of RP at the time of $\mathrm{NC}$ was 6.9 years in the group who developed a CTD and 6.5 years in the group who did not. In patients who developed a CTD during the follow-up, duration of RP up to 49 years has been reported. Our data suggest that the duration of RP is a very poor criterion for identifying patients at risk for the development of secondary RP.

The mean age was not different in the two patient groups, although children and very young adults (age between 10 and 21 ) were only seen in the group who developed a CTD during the follow-up. The number might be too low for a conclusion, but it suggests that in children and young adults with RP and pathological capillaries the risk for an underlying disease is higher.

In 201 patients $(77.1 \%$ female) the diagnosis of an underlying disease had already been made at the time of NC. About half of the patients had been diagnosed with a form of Scl; in these patients a microangiopathy in NC was present in over $80 \%$. In contrast, only in $37.9 \%$ of patients with SLE was microangiopathy present. Pathological capillary morphology has been reported in 2 $90 \%$ of SLE patients $[10,31]$. This variation in published numbers might be due, at least in part, to the definition of microangiopathy used by the authors. Furthermore, it has been speculated that the presence of microangiopathy might be associated with clinical features, such as RP or specific antibodies. Furtado et al. [32] found a significant association between microangiopathy in SLE and RP, and similar findings were reported by Caspary et al. [33]; however, in a study of 51 patients with SLE Bongard et al. [34] found no correlation between abnormal capillaroscopic findings and RP. In our study, only patients with RP were included, which might be one reason for the rather high incidence of microangiopathy in our group. A higher incidence of microangiopathy among SLE patients with positive anticardiolipin and anti-Ul ribonucleoprotein antibodies has been demonstrated, suggesting direct damage to the endothelium by these antibodies [32, 34]. However, specific autoantibodies in Scl patients (i.e. anti-Scl-70 and anticardiolipin antibody) do not seem directly linked to the expression of a singular capillaroscopic pattern [35].

In conclusion, in patients presenting with RP the presence of either giant capillaries, avascular fields or irregular architecture in NC is predictive for the development of a CTD, mainly scleroderma, CREST and MCTD. NC may not be a valuable diagnostic tool in SLE, but it might be helpful in identifying a subgroup of patients with different evolution and prognosis of the disease. The duration of RP was not a criterion for the risk of future development of CTD. Despite the fact that in $\mathrm{Scl}$ a specific diffusion pattern after $\mathrm{NaF}$ injection might be present, the additional use of $\mathrm{NaF}$ does not facilitate early detection of a CTD.

\section{Take home message}

In patients with RP and negative serological tests, the presence of giant capillaries, avascular fields or irregular architecture in $\mathrm{NC}$ is predictive for the development of a CTD, mainly scleroderma, CREST and MCTD. Based on our data the duration of RP is not a criterion for the risk of future development of CTD.

\section{References}

1. Maricq HR, Carpentier PH, Weinrich MC, Keil JE, Franco A. Drouet P, Poncot OC, Maines MV (1993) Geographic variation in the prevalence of Raynaud's phenomenon: Charleston, SC, USA, vs. Tarentaise, Savoie, France. J Rheumatol 20:7076

2. Kallenberg CG, Wouda AA, Hoet MH, van Venrooij WJ (1988) Development of connective tissue disease in patients presenting with Raynaud's phenomenon: a six year follow up with emphasis on the predictive value of antinuclear antibodies as detected by immunoblotting. Ann Rheum Dis 47:634-641

3. Bennett R, Bluestone R, Holt PJ, Bywaters EG (1971) Survival in scleroderma. Ann Rheum Dis 30:581-588

4. Tuffanelli DL, Winkelmann RK (1961) Systemic scleroderma, a clinical study of 727 cases. Arch Dermatol 84:359-371

5. Fessel WJ (1974) Systemic lupus erythematosus in the community. Incidence, prevalence, outcome, and first symptoms; the high prevalence in black women. Arch Intern Med 134:1027-1035

6. Kallenberg CG, Wouda AA, The TH (1980) Systemic involvement and immunologic findings in patients presenting with Raynaud's phenomenon. Am J Med 69:675-680

7. Wollersheim H, Thien T, Hoet MH, Van Venrooy WJ (1989) The diagnostic value of several immunological tests for antinuclear antibody in predicting the development of connective tissue disease in patients presenting with Raynaud's phenomenon. Eur J Clin Invest 19:535-541

8. Weiner ES, Hildebrandt S, Senecal JL, Daniels L, Noell S, Joyal F, Roussin A, Earnshaw W, Rothfield NF (1991) Prognostic significance of anticentromere antibodies and anti-topoisomerase I antibodies in Raynaud's disease. A prospective study. Arthritis Rheum 34:68-77

9. Cutolo M, Sulli A, Pizzorni C, Accardo S (2000) Nailfold videocapillaroscopy assessment of microvascular damage in systemic sclerosis. J Rheumatol 27:155-160

10. Maricq HR, LeRoy EC, D'Angelo WA, Medsger TA Jr, Rodnan GP, Sharp GC, Wolfe JF (1980) Diagnostic potential of in vivo capillary microscopy in scleroderma and related disorders. Arthritis Rheum 23:183-189

11. Lee P, Sarkozi J, Bookman AA, Keystone EC, Armstrong SK (1986) Digital blood flow and nailfold capillary microscopy in Raynaud's phenomenon. I Rheumatol 13:564-569

12. Grassi W, Medico PD, Izzo F, Cervini C (2001) Microvascular involvement in systemic sclerosis: capillaroscopic findings. Semin Arthritis Rheum 30:397-402

13. Zufferey P, Depairon M, Chamot AM, Monti M (1992) Prognostic significance of nailfold capillary microscopy in patients with Raynaud's phenomenon and scleroderma-pattern abnormalities. A six-year follow-up study. Clin Rheumatol 11:536-541

14. Passiu G, Sebastiani GD, Galeazzi M, Tuveri MA, Nicosia PM, Boirivant R (1990) Prognostic factors in Raynaud's phenomenon: usefulness of antinuclear antibodies and of periungual capillaroscopy. Medicina (Firenze) 10:405-407

15. Cutolo M, Grassi W, Matucci Cerinic M (2003) Raynaud's phenomenon and the role of capillaroscopy. Arthritis Rheum $48: 3023-3030$ 
16. Brulisauer M, Bollinger A (1991) Measurement of different human microvascular dimensions by combination of videomicroscopy with $\mathrm{Na}$-fluorescein $(\mathrm{NaF})$ and indocyanine green (ICG) in normals and patients with systemic sclerosis. Int J Microcirc Clin Exp 10:21-31

17. Moneta G, Vollenweider U, Dubler B, Bollinger A (1986) Diagnostic value of capillaroscopy with and without fluorescent dyes to detect early connective tissue disease. Vasa 15:143-149

18. Bollinger A, Fagrell B (1990) Clinical capillaroscopy. Hogrefe, Göttingen

19. Schmidt JA, Caspary L, von Bierbrauer A, Ehrly AM, Junger M, Jung F, Lawall H (1997) Standardization of nailfold capillary microscopy in routine diagnosis. Vasa $26: 5-10$

20. Masi AT (1988) Classification of systemic sclerosis (scleroderma): relationship of cutaneous subgroups in early disease to outcome and serologic reactivity. J Rheumatol 15:894-898

21. Alarcon-Segovia D, Cardiel MH (1989) Comparison between 3 diagnostic criteria for mixed connective tissue disease. Study of 593 patients. J Rheumatol 16:328-334

22. Bohan A, Peter JB (1975) Polymyositis and dermatomyositis (second of two parts). N Engl J Med 292:403-407

23. Vitali C, Bombardieri S, Jonsson R, Moutsopoulos HM Alexander EL, Carsons SE, Daniels TE, Fox PC, Fox RI, Kassan SS et al (2002) Classification criteria for Sjogren's syndrome: a revised version of the European criteria proposed by the American-European Consensus Group. Ann Rheum Dis 61:554-558

24. Kenik JG, Maricq HR, Bole GG (1981) Blind evaluation of the diagnostic specificity of nailfold capillary microscopy in the connective tissue diseases. Arthritis Rheum 24:885-891

25. Maricq HR, LeRoy EC (1973) Patterns of finger capillary abnormalities in connective tissue disease by "wide-field" microscopy. Arthritis Rheum 16:619-628

26. Houtman PM, Kallenberg CG, Fidler V, Wouda AA (1986) Diagnostic significance of nailfold capillary patterns in patients with Raynaud's phenomenon. An analysis of patterns discriminating patients with and without connective tissue disease. J Rheumatol 13:556-563

27. Bollinger A, Jager K, Siegenthaler W (1986) Microangiopathy of progressive systemic sclerosis. Evaluation by dynamic fluorescence videomicroscopy. Arch Intern Med 146:1541-1545

28. Monticone G, Colonna L, Palermi G, Bono R, Puddu P (2000) Quantitative nailfold capillary microscopy findings in patients with acrocyanosis compared with patients having systemic sclerosis and control subjects. J Am Acad Dermatol 42:787-790

29. Jacobs MJ, Breslau PJ, Slaaf DW, Reneman RS, Lemmens JA (1987) Nomenclature of Raynaud's phenomenon: a capillary microscopic and hemorheologic study. Surgery 101:136-145

30. LeRoy EC, Medsger TA Jr (1992) Raynaud's phenomenon: a proposal for classification. Clin Exp Rheumatol 10:485-488

31. Redisch W, Messina EJ, Hughes G, McEwen C (1970) Capillaroscopic observations in rheumatic diseases. Ann Rheum Dis 29:244-253

32. Furtado RN, Pucinelli ML, Cristo VV, Andrade LE, Sato EI (2002) Scleroderma-like nailfold capillaroscopic abnormalities are associated with anti-U1-RNP antibodies and Raynaud's phenomenon in SLE patients. Lupus 11:35-41

33. Caspary L, Schmees C, Schoetensack I, Hartung K, Stannat S, Deicher H, Creutzig A, Alexander K (1991) Alterations of the nailfold capillary morphology associated with Raynaud phenomenon in patients with systemic lupus erythematosus. J Rheumatol 18:559-566

34. Bongard $\mathrm{O}$, Bounameaux $\mathrm{H}$, Miescher PA, De Moerloose $\mathrm{P}$ (1995) Association of anticardiolipin antibodies and abnormal nailfold capillaroscopy in patients with systemic lupus erythematosus. Lupus 4:142-144

35. Cutolo M, Pizzorni C, Tuccio M, Burroni A, Craviotto C, Basso M, Seriolo B, Sulli A (2004) Nailfold videocapillaroscopic patterns and serum autoantibodies in systemic sclerosis. Rheumatology (Oxford) 43:719-726 